Objective radiographic responses were observed in $53 \%$ of patients...

\title{
Matching mutations to immunotherapy response
}

The presence of mismatch repair (MMR) deficiency could be used as a predictor of response of solid tumours to PD-1 (programmed cell death protein 1) blockade immunotherapy, according to a report published in Science. The clinical trial, which is still ongoing, showed that a wide range of cancer types with MMR deficiency responded to PD-1 blockade, including a number of gastrointestinal malignancies such as colorectal cancer and pancreatic cancer.

MMR-deficient cancers are predicted to have a large number of mutationassociated neoantigens that could be recognized by the immune system. An initial proof-of-concept study previously showed that colorectal cancers with MMR deficiency were sensitive to PD-1 immune checkpoint blockade. In the new trial, Le et al. expanded upon this initial study and evaluated the efficacy of PD-1 inhibition in patients $(n=86)$ with advanced MMR-deficient cancer across 12 different tumour types. Effectiveness was tested in a number of gastrointestinal cancers, among others, including colorectal, pancreatic and gastro-oesophageal tumours.

Radiographic responses, progression-free survival (PFS) and overall survival (OS) were measured using RECIST v1.1 criteria. Objective radiographic responses were observed in $53 \%$ of patients ( 46 of 86 ), with $21 \%$ (18 of 86 ) achieving a complete response. Notably, the objective response rate was similar between colorectal cancer and other cancer types. The study is ongoing and, as yet, the median PFS and median OS has not been reached. Adverse events during treatment were similar to previous reports, with the majority of patients (74\%) having experienced one, although most were low-grade and manageable.

The researchers also tested the hypothesis that checkpoint blockade induces immune responses, particularly peripheral expansion of tumour-specific $T$ cells, in reaction to mutationassociated neoantigens. Examining T-cell receptor clonal dynamics and recognition of mutation-associated neoantigens in patients responding to PD-1 inhibition, they observed rapid expansion of neoantigen-specific T-cell clones that were reactive to mutant neopeptides in tumours. These clones were present at very low frequency before treatment, but rapidly increased after initiation of treatment.

"In the future, testing for MMR deficiency in patients who are refractory to other treatments might be considered in order to identify those who may benefit from PD-1 pathway blockade, regardless of tumour type," write the authors.

Katrina Ray

ORIGINAL ARTICLE Le, D. T. et al. Mismatch repair deficiency predicts response of solid tumors to PD-1 blockade. Science 357, 409-413 (2017) 